\title{
Sonographic Features of Borderline Phyllodes Tumor of the Breast: A Case Report
}

\author{
Yaqin Sun, MD ${ }^{a}$, Shumin Wang, MD, PhD, ${ }^{b, *}$, Zhanfei Zhe, MD ${ }^{a}$, Xin Qi, MD ${ }^{a}$, Li Li, MD ${ }^{a}$, Qin Liu, MD ${ }^{a}$ \\ ${ }^{a}$ Ordos Center Hospital, Ordos, Inner Mongolia, China; ${ }^{b}$ Department of Ultrasound, Peking University Third Hospital, Beijing, China \\ Received February 19, 2021; revision received April 20, 2021; accepted April 25, 2021
}

Abstract: Phyllodes tumors of the breast are rare fibroepithelial neoplasm which are generally divided into benign, borderline, or malignant. In this case study, we reported a 40-year-old female presented with an asymptomatic right breast mass underwent sonographic evaluation. The surgical histopathology was diagnosed as borderline phyllodes tumor. Sonographic features of the tumor are described in detail, and the relevant literatures are reviewed and discussed.

Key words: Phyllodes tumor; Breast; Sonography

Advanced Ultrasound in Diagnosis and Therapy 2021; 03: 262-264

DOI: 10.37015/AUDT.2021.210006

$\mathrm{B}$ reast phyllodes tumors (PTs) is a very rare fibroepithelial neoplasm, accounting for 0.3$1.0 \%$ of breast tumors [1-3]. PTs are classified histologically as benign, borderline, or malignant according to the presence of tumor margins, cellular atypia, mitotic activity, and overgrowth in the stroma [46]. PTs have a high recurrence rate and may transform into malignant disease, leaving no margin for errors in the initial diagnosis [7]. In the following case, we report a breast borderline phyllodes tumor in a middle-aged female presented with the enlargement of the breast mass for 1 month.

\section{Case Report}

Patient was a 40-year-old female. Although having noticed a mass in her right breast three years ago, she had not sought medical attention until the mass growned rapidly within one month period.

Clinical examination palpated a soft mass with a clear margin in upper external quadrant of the right breast, which covered with cyan skin, measured approximately $2.0 \mathrm{~cm}$ in diameter. There were not palpable lymph nodes in the axillary and other superficial areas. Her history report shown that she had a lumpectomy on her right breast, and the pathological report diagnosed as a fibroadenoma. There was no relevant past medical history and no family history of breast or ovarian cancer.

Ultrasound examination showed a lobulated hypoechoic and no circumscribed margin mass $(27 \times 22$ $\times 26 \mathrm{~mm}$ ) with posterior acoustic enhancement. There were multiple cystic areas seen within the tumor (Fig.1). Color Doppler examination revealed a high-impedance pulsatile signal within the mass (Fig.2). The patient was treated with wide local excision. In surgically excised specimen, tissue found an indented friable and soft mass inside several cysts(Fig.3). Photomicrograph showed a borderline PT (Fig.4A and 4B).

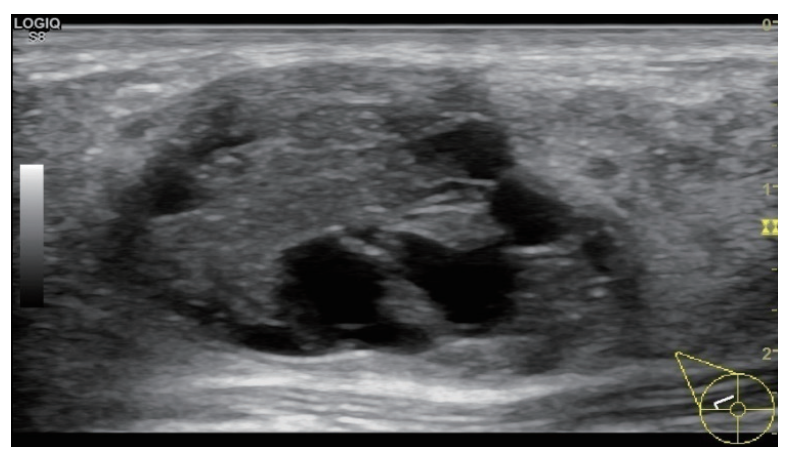

Figure 1 Ultrasound imaging of the breast showed a lobulated hypoechoic and no circumscribed margin mass with posterior acoustic enhancement and cystic areas within the mass.

* Corresponding author: Department of Ultrasound, Peking University Third Hospital, 49 Huayuan North Road, Haidian District, Beijing, 100191, China

e-mail: shuminwang2014@163.com unrestricted use, distribution and reproduction in any medium provided that the original work is properly attributed. 


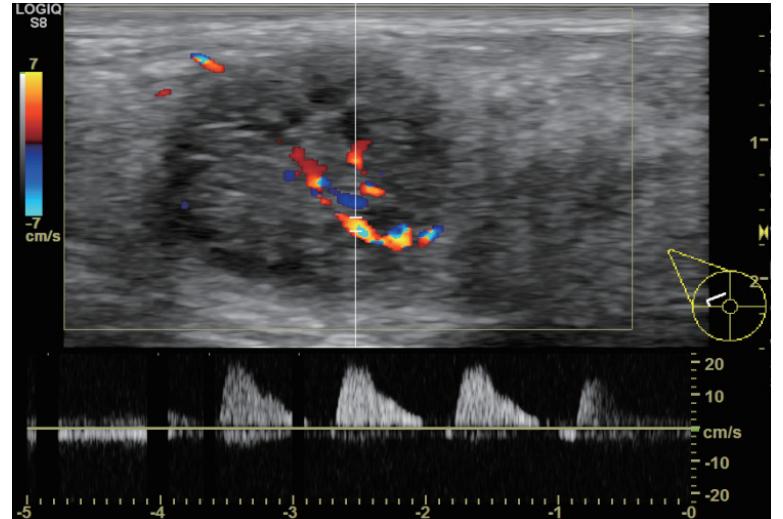

Figure 2 Color Doppler flow imaging revealed a high-impedance pulsatile signal within the tumor.

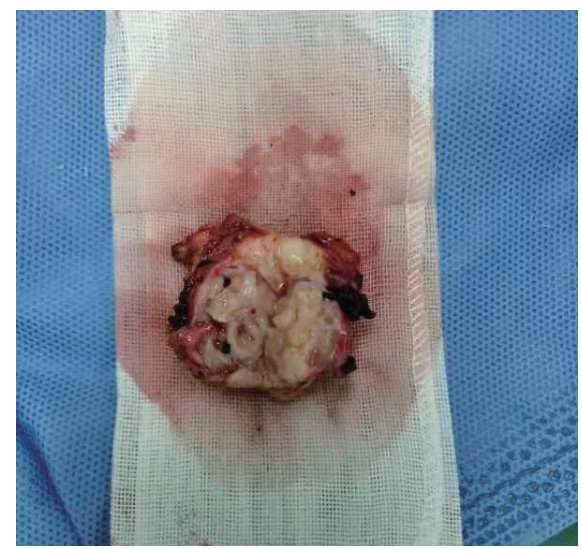

Figure 3 Macroscopic examination of the ablated tissue revealed an indented friable and soft mass inside several cysts.
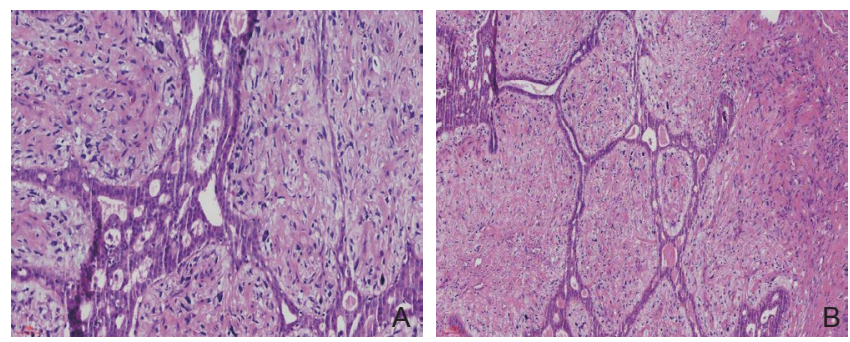

Figure 4 Photomicrographic inspection revealed a borderline phyllodes tumor with increase in stromal cellularity, moderate atypia, and occasional mitoses. Hematoxylin-eosin staining: (A) Magnification, 40×; (B) Magnification, $\times 20$

Nine months later, additional solid cystic nodule with measurement of $15 \times 12 \times 7 \mathrm{~mm}$ was identified at the incision of the right breast, and color Doppler imaging showed a few blood flows signal from interior of the nodule (Fig.5). No abnormally swollen lymph nodes were observed under the axillary region. Based on clinical history and ultrasound findings, the recurrence of lobular tumor was considered. The patient underwent surgical intervention for total right breast resection. Postoperative pathology confirmed the lesion as low to severe malignant lobular tumor.

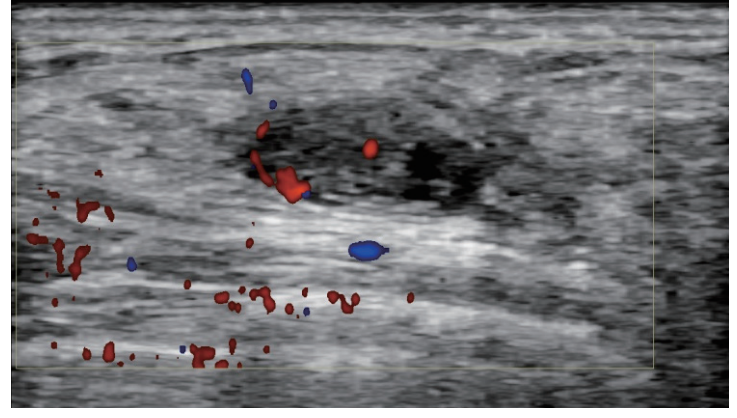

Figure 5 Color Doppler examination of recurrent mass revealed a few blood flow signals within the nodule. Surgical pathology confirmed the lesion as low to severe malignant lobular tumor.

\section{Discussion}

PTs are uncommon fibroepithelial neoplasms that account for $<1 \%$ of all mammary tumors. The World Health Organization classified PTs into benign, borderline, or malignant categories based on features of the stromal component [8]. Although most of PTs tend to behave in a benign fashion clinically, the risk of local recurrences and distant metastases can occur sometimes. The accurate identification of PTs preoperatively is important to make surgical planning, in order to avoid operative complications due to inadequate excision or surgical overtreatment. Strodeet al. [9] reported that a fast-growing benign appearing mammary mass in a female more than 35 years old needed to warrant concern for a PT and further investigation. Therefore, rapidly growing breast mass is the most common reason for patients to visit the hospital, as presented in our case. However, the growth rate has not been definitely associated with malignancy.

As approximately $20-30 \%$ [10] of resected PTs are malignant and about $25 \%$ [11] of malignant PTs metastasize. Thus, PTs must be treated surgically. Moreover, wide excision of phyllodes tumors with adequate margins is essential to the prevention of local recurrence and to provide an accurate diagnosis as to whether it is benign, borderline, or malignant [12]. Therefore, the accurate diagnosis of the PTs is critical and important for clinical management.

Despite the prognostic differences between benign and borderline or malignant phyllodes tumors, the imaging features of differing histologic subtypes have little attention [13]. There are five clinical and ultrasound features that may aid in distinguishing benign from borderline or malignant phyllodes tumor types. At first, the size of the tumor needs to be considered. Liberman et al. [3] noted that phyllodes tumors larger than $3 \mathrm{~cm}$ had a higher likelihood of being malignant. Kalamboet al. [13] found that the tumor size greater than $7 \mathrm{~cm}$ is indicative of an increased likelihood of a borderline or malignant phyllodes tumor. In our case, the tumor was less than 
$3 \mathrm{~cm}$ so that the tumor size may not be a characteristic indicator of malignant phyllodes tumors. Certainly, further study with more cases need to support this view.

Secondly, a large, lobulated, solid breast mass with intramural cysts and multiple septations is sonographic feature for phyllodes tumors which was observed in our case. In the study by Duman et al. [12] phyllodes tumors showed the presence of internal cystic areas was considerably different between fibroadenomas and phyllodes tumors. However, Harper et al. [14] showed that intramural cysts suggesting focal necrosis or degeneration are not pathognomonic for phyllodes tumors, which can occasionally be observed in other well-circumscribed tumors, such as medullary carcinoma or fibroadenoma. Consequently, the reliability of intramural cysts and septations in the diagnosis of phyllodes tumors requires further study.

Thirdly, the posterior acoustic enhancement is generally one of the sonographic characteristics to differentiate between benign and malignant breast tumors $[15,16]$. The posterior acoustic enhancement of the tumor is another feature appearance in our case. In accordance with the literature, posterior acoustic enhancement was more commonly detected in phyllodes tumors than in fibroadenomas [12,17,18]. However, Chao et al. [19] suggested that the tumor posterior acoustic phenomenon does not characterize phyllodes tumors and it is not a significant feature to distinguish benign from malignant phyllodes tumors.

Fourthly, the absence of microcalcifications is a characteristic sonographic feature to distinguish between phyllodes tumors and fibroadenoma [12,17,19], as presented in our case. Finally, color Doppler examination revealed a high-impedance pulsatile hypervascularity signal in our case, which is in accordance with the findings in the literature [12]. This finding suggested that hypervascularity might be a significant feature of phyllodes tumors while fibroadenomas are more frequently hypovascular lesions.

\section{Conclusion}

In conclusion, phyllodes tumors pose a unique challenge to the radiologist because they can be difficult to distinguish from other benign and malignant tumors of the breast. When a patient presents with a palpable rapidly growing, painless mass and sonographic imaging demonstrates irregular and no circumscribed margins solid mass with intramural cysts, phyllodes tumors should be considered and excisional biopsy should be performed to establish an accurate diagnosis for clinical management.

\section{Acknowledgements}

This work was supported by the Inner Mongolia Natural Science Foundation 2020MS080881 and Ordos Applied Technology Research and Development Fund Plan [2018]516.

\section{Conflict of interest}

The authors have no conflict of interest to declare.

\section{References}

[1] Palmer ML, De Risi DC, Pelikan A, Patel J, Nemoto T, Rosner D, et al. Treatment options and recurrence potential for cystosarcoma phyllodes. Surg Gynecol Obstet 1990; 170:193-196.

[2] Rowell MD, Perry RR, Hsiu JG, Barranco SC. Phyllodes tumors. Am J Surg 1993; 165:376-379.

[3] Liberman L, Bonaccio E, Hamele-Bena D, Abramson AF, Cohen MA, Dershaw DD. Benign and malignant phyllodes tumors: mammographic and sonographic findings. Radiology 1996; 198:121-124.

[4] Lakhani S R. WHO Classification of Tumours of the Breast. International Agency for Research on Cancer, 2012.

[5] Tan PH, Jayabaskar T, Chuah KL, Lee HY, Tan Y, Hilmy M, et al. Phyllodes tumors of the breast: the role of pathologic parameters. $\mathrm{Am}$ J Clin Pathol 2005; 123:529-540.

[6] Böcker W. WHO-Klassifikation der Tumoren der Mamma und des weiblichenGenitale: Pathologie und Genetik. WHO classification of breast tumors and tumors of the female genital organs: pathology and genetics. Verh Dtsch Ges Pathol. 2002; 86:116-9.

[7] Eroglu E, Irkkan C, Ozsoy M, Eroglu F. Phyllodes tumor of the breast: case series of 40 patients. Eur J Gynaecol Oncol 2004; 25:123-125.

[8] Belkacémi Y, Bousquet G, Marsiglia H, Ray-Coquard I, Magné N, Malard Y, et al. Phyllodes tumor of the breast. Int J Radiat Oncol Biol Phys 2008; 70:492-500.

[9] Strode M, Khoury T, Mangieri C, Takabe K. Update on the diagnosis and management of malignant phyllodes tumors of the breast. Breast 2017; 33:91-96.

[10] Lee S, Nodit L. Phyllodes tumor of vulva: a brief diagnostic review. Arch Pathol Lab Med 2014;138(11):1546-1550.

[11] Reinfuss M, Mituś J, Duda K, Stelmach A, Ryś J, Smolak K. The treatment and prognosis of patients with phyllodes tumor of the breast: an analysis of 170 cases. Cancer 1996; 77:910-916.

[12] Duman L, Gezer NS, Balcı P, Altay C, Başara I, Durak MG, et al. Differentiation between phyllodes tumors and fibroadenomas based on mammographic sonographic and MRI features. Breast Care (Basel) 2016; 11:123-127.

[13] Kalambo M, Adrada BE, Adeyefa MM, Krishnamurthy S, Hess K, Carkaci S, et al. Phyllodes tumor of the breast: ultrasound-pathology correlation. AJR Am J Roentgenol 2018;210: W173-W179.

[14] Harper AP, Kelly-Fry E, Noe JS, Bies JR, Jackson VP. Ultrasound in the evaluation of solid breast masses. Radiology 1983; 146:731-736.

[15] Skaane P, Engedal K. Analysis of sonographic features in the differentiation of fibroadenoma and invasive ductal carcinoma. AJR Am J Roentgenol 1998; 170:109-114.

[16] Chao TC, Lo YF, Chen SC, Chen MF. Prospective sonographic study of 3093 breast tumors. J Ultrasound Med 1999; 18:363-372.

[17] Yilmaz E, Sal S, Lebe B. Differentiation of phyllodes tumors versus fibroadenomas. Acta Radiol 2002; 43:34-39.

[18] Bode MK, Rissanen T, Apaja-Sarkkinen M. Ultrasonography and core needle biopsy in the differential diagnosis of fibroadenoma and tumor phyllodes. Acta Radiol 2007; 48:708-713.

[19] Chao TC, Lo YF, Chen SC, Chen MF. Sonographic features of phyllodes tumors of the breast. Ultrasound Obstet Gynecol 2002; 20:64-71 